\title{
Interdiffused AlGaAs-GaAs Quantum Well for Improved Electroabsorptive Modulation
}

\author{
Wallace C. H. Choy and E. Herbert Li, Senior Member, IEEE
}

\begin{abstract}
This is a theoretical study of the effects of two asgrown structural parameters on the modulation properties of $\mathbf{A l} \mathbf{l}_{x} \mathbf{G a}_{1-x} \mathbf{A s}-\mathbf{G a A s}$ quantum wells (QW's), which are the Al concentration in barrier and the thickness of the well layer serving as initial conditions before interdiffusion. The results show that, with a larger Al concentration and a wider well width, the range of interdiffusion for an enhanced electroabsorption (EA) change increases with both of these parameters, while insertion loss increases with the former and decreases with the latter. However, the increase in loss is lower than that of the rectangular QW for the same magnitude of absorption change. The range of a tunable absorption-peak wavelength produced by interdiffussion increases with increasing Al concentration and decreases with increasing well width. Moreover, in a moderately interdiffused $\mathrm{QW}$, the required bias reduces for the same level of EA modulation. For the best device operation, interdiffused QW's with the Al concentration between 0.3 and 0.4 and well width between 10 and $12 \mathrm{~nm}$ are most suitable for developing a general-purpose electroabsorptive modulator. When applied in high-speed modulators, the EA of a wide and shallow QW active-region structure can be further enhanced by the use of corresponding interdiffusion.
\end{abstract}

Index Terms - Diffusion process, electroabsorption, intermixing quantum well, modeling, optical materials/devices, quantumconfined Stark effect, quantum-well devices, tunable devices.

\section{INTRODUCTION}

$\mathbf{T}$ THE QUANTUM well (QW) that has been actively investigated and successfully demonstrated in waveguide and vertical-cavity types of modulator is the $\mathrm{Al}_{x} \mathrm{Ga}_{1-x} \mathrm{As}-\mathrm{GaAs}$ QW [1]. This is due to their advanced properties including a sharp exciton peak and a large electroabsorption (EA) change. The QW EA modulator has been considered recently as an important component in the development of high speed (40$\mathrm{GHz}$ modulation bandwidth) optical communication systems [2]. A tailorable material system (in terms of its optical properties) is important to the improved performance of a modulator because the quality of the QW modulation depends critically on the material properties. These properties include field induced EA change $\Delta \alpha$, contrast ratio (CR), Stark shift,

Manuscript received March 10, 1997; revised October, 14, 1997. This work was supported by the RGC earmarked grant and the University of Hong Kong CRCG grant, and by the Croucher Foundation.

W. C. H. Choy was with the Department of Electrical and Electronic Engineering, University of Hong Kong, Hong Kong. He is now with the School of Electronic Engineering, Information Technology, and Mathematics, University of Surrey, Guildford, Surrey GU2 5XH, U.K.

E. H. Li is with the Department of Electrical and Electronic Engineering, University of Hong Kong, Hong Kong.

Publisher Item Identifier S 0018-9197(98)04564-3. residual loss $\alpha_{\text {loss }}$ (which is considered to be the background absorption of the active region), voltage swing, and chirping.

Recently, the AlGaAs-GaAs interdiffused or intermixed QW (DFQW) structures have also been actively investigated and widely used in several optical devices, such as EA waveguides [3] and vertical-cavity [4], [5] types of modulator, lateral confinement waveguides [6], and wavelength tunable lasers [7], [8]. DFQW has even been regarded as a key candidate in the development of optoelectronic integration [9]. However, there is no study on the effects of the variation in the two initial conditions prior to thermal diffusion, such as asgrown $\mathrm{Al}$ concentration of barrier and thickness of well width. The knowledge of this is crucial to the optimal performance of the DFQW modulators. This motivates the present theoretical analysis in the interdiffused $\mathrm{Al}_{x} \mathrm{Ga}_{1-x} \mathrm{As}-\mathrm{GaAs}$ QW toward the development of an improved performance EA-modulator.

In this paper, we theoretically study the $\mathrm{Al}_{x} \mathrm{Ga}_{1-x} \mathrm{As}-\mathrm{GaAs}$ DFQW's with modified optical properties which are developed from different as-grown conditions rectangular QW's. In Section II, the computational consideration of the material system is discussed. In Section III, the results are discussed in two foci: one on the confined eigenstates and the other on the optical modulation properties. In each of these, the typical case of $x=0.3$ and as-grown well width $L_{z}$ of $10 \mathrm{~nm}$ is addressed first and followed by the discussion of the effects of the two initial conditions, i.e., the $\mathrm{Al}$ concentration and as-grown well width. Section IV provides a summary of the modified EA properties cased by these two conditions. In Section $\mathrm{V}$, the advantage of employing DFQW with different initial conditions as the active-region material in EA-modulator is discussed. Finally, the conclusion will be drawn in Section IV.

\section{COMPutational CONSIDERATions}

The interdiffusion of the QW composition profile is described by an error function profile [10]. The extent of the interdiffusion process is characterized by a diffusion length $L_{d}$, which is defined as $L_{d}=(D t)^{1 / 2}$, where $D$ and $t$ are the interdiffusion coefficient and the annealing time, respectively. ${ }^{1}$ The square of this length represents half of the variance of the interdiffusion distribution in a linear flow situation (i.e., $L_{d}^{2}=1 / 2$ variance, and $L_{d}=$ standard deviation $/ \sqrt{2}$ ). The as-grown $\mathrm{QW}$ is defined as $L_{d}=0$. The diffused $\mathrm{Al}$ composition profiles $\tilde{w}(z)$ across the $\mathrm{QW}$ structure is given

\footnotetext{
${ }^{1}$ It should be noted here that the definition of $L_{d}$ varies in the literature; for instance, some authors define $L_{d}$ as $2(D t)^{1 / 2}$, i.e., $L_{d}^{2}=2 \times$ variance and $L_{d}=\sqrt{2} \times$ standard deviation.
} 
by

$$
\tilde{w}(z)=w_{0}\left\{1-\frac{1}{2}\left[\operatorname{erf}\left(\frac{L_{z}+2 z}{4 L_{d}}\right)+\operatorname{erf}\left(\frac{L_{z}-2 z}{4 L_{d}}\right)\right]\right\}
$$

where $w_{0}$ is the as-grown $\mathrm{Al}$ mole fraction in the barrier, $L_{z}$ is the as-grown well width of the $\mathrm{QW}, z$ is both the quantization and the growth axis (QW centered at $z=0$ ), and erf denotes the error function. The interdiffusion-induced nonlinear confinement profile $U_{r}(z)$ is defined as

$$
U_{r}(z)=Q_{r}\left[\tilde{E}_{g}(z)-\tilde{E}_{g}(z=0)\right]+z e F
$$

where the subscript $r$ denotes either the electrons in the conduction band or heavy and light holes in the valence band. The bandgap $\tilde{E}_{g}(z)$ equals $E_{g}[w=\tilde{w}(z)]$, and $Q_{r}$ is the band offset splitting. The additional term $z e F$ is the effect of applied field on the QW structure, and $e$ is the electron charge. The subband structures are calculated numerically using spatially dependent effective masses, conduction band nonparabolicity, and valence subband mixing. Details of these subband calculations are discussed in [12]. The field effect on the band-edge eigenstates is calculated according to a scheme developed by Bloss [11]. These subbands are then used to calculate the heavy hole $(\mathrm{HH})$ and light hole $(\mathrm{LH})$ related $1 \mathrm{~S}$ exciton-binding energies and wavefunctions by a perturbative-variational method [13].

The imaginary part of the dielectric function for the $\Gamma$ valley, $\varepsilon_{2}^{\Gamma}$, is calculated as [5]

$$
\varepsilon_{2}^{\Gamma}(\omega)=\varepsilon_{2}^{\mathrm{exc}}(\omega)+\varepsilon_{2}^{\text {bound }}(\omega)+\varepsilon_{2}^{\mathrm{con}}(\omega)
$$

where $\omega$ is the angular frequency, $\varepsilon_{2}^{\text {exc }}$ is the $1 \mathrm{~S}$ exciton contribution derived by the density-matrix approach at the subband edge without the influence of band mixing, and $\varepsilon_{2}^{\text {bound }}$ is conduction-valence band bound-state contribution without the electron-hole interaction. $\varepsilon_{2}^{\text {con }}$ is the contribution from the unbound continuum states above the barrier. The $\Gamma$-valley contribution to $\varepsilon_{2}^{\text {con }}$ is calculated for over 40 states (for each of the bands) above the barrier and then combined with the bulk contribution [14].

The polarization-dependent absorption coefficient $\alpha^{T}(\omega)$ is the sum of $\alpha^{\text {bound }}(\omega), \alpha^{\operatorname{exc}}(\omega)$, and $\alpha^{\operatorname{con}}(\omega)$. They are the absorption coefficient of the bound state, the exciton state, and the continuum region, respectively. These different parts of the absorption coefficient can be calculated from the corresponding parts of the dielectric functions in $\varepsilon_{2}^{\Gamma}(\omega)$ using the relation in

$$
\alpha^{\gamma}(\omega)=\left[\omega \varepsilon_{2}^{\gamma}(\omega)\right] /\left[c_{0} n_{r}(\omega)\right]
$$

where the superscript $\gamma$ denotes either the bound state, the exciton state, or the continuum region. $c_{0}$ and $n_{r}(\omega)$ are the speed of light in vacuum and the refractive index of the DFQW, respectively. The field-induced change of absorption coefficient $\Delta \alpha(w)$ can then be calculated by

$$
\Delta \alpha(\omega)=\alpha^{T}(\omega)_{F \neq 0}-\alpha^{T}(\omega)_{F=0} .
$$

The contrast ratio for a modulator is defined as the relative optical intensity modulation and is given as [15]

$$
\mathrm{CR}(\mathrm{dB})=10 \log \left[\frac{\exp \left(-\alpha_{\mathrm{ON}} \ell\right)}{\exp \left(-\alpha_{\mathrm{OFF}} \ell\right)}\right]
$$

where $\alpha_{\mathrm{ON}}$ and $\alpha_{\mathrm{OFF}}$ are the absorption in $\mathrm{ON}$ and $\mathrm{OFF}$ states, respectively, and $\ell$ is the modulation length.

\section{Results AND Discussions}

In order to study the effects of the variations in the two initial conditions, as-grown aluminum concentration in barrier and thickness of well width, combinations of different $\mathrm{Al}$ concentrations $(x=0.2,0.3$, and 0.4$)$ and well widths ( $L_{z}=$ 8,10 , and $12 \mathrm{~nm}$ ) are investigated.

The as-grown structure of $x=0.3$ and $L_{z}=10 \mathrm{~nm}$ is considered to be typical and is discussed first. Through the understanding of the DFQW of the typical structure, the effects of the two initial conditions are then analyzed. The independent effect of the aluminum concentration is studied by minimizing the effect of the as-grown well width, which is analyzed to $L_{z}=12 \mathrm{~nm}$. On the other hand, the effect of as-grown well width is investigated by using $x=0.3$. This is because with $x=0.2$, only weak applied fields can be supported whilst with $x=0.4$, the $\mathrm{Al}$ concentration is so high that it brings an overwhelming effect on the DFQW. Thus, the width effect cannot be shown intuitively.

In the calculation of absorption coefficient, the Lorentzian broadening factors half-width-half-maximum (HWHM) are extracted from experimental data [16], [17]. The HWHM of the heavy hole $(\mathrm{HH})$ and the light hole $(\mathrm{LH})$ are considered to be the same and have a value of $3 \mathrm{meV}$ which is averaged from the values taken from [16] and [17]. It should be noted that although interdiffusion will enhance the broadening factor [18], its relation is not well known and is therefore considered to be a constant here.

Since an EA modulator operates at the wavelength of the respective biased band-edge exciton peak [19], it is important to understand the QW eigenstate properties and their effect on the modulation performance including the absorption change $\Delta \alpha$, Stark shift, residual loss $\alpha_{\text {loss }}$, operation wavelength $\lambda_{\text {op }}$ and the required bias. It should be noted that we restrict our discussion to the first eigenstates in the conduction and valence bands, where they are denoted as $\mathrm{C} 1$ and $\mathrm{HH} 1$, respectively. Also, the interdiffusion extent of $L_{d}=4 \mathrm{~nm}$ is considered to be extensive.

\section{A. Interdiffusion Contribution to a $Q W$ Confinement}

In an interdiffused QW, the confinement profile is graded in nature with no well-defined well width and well depth. Therefore, the following analysis will be based on a changeable well potential depth (under no bias), an effective width of QW at the first eigenstate energy and its wavefunction. Generally, both a deeper well depth and a narrower well width enhance the confinement of carriers in QW.

In the typical unbiased QW structure, the effective width reduces by $40 \%$ for $L_{d} \leq 2 \mathrm{~nm}$, while the potential depth remains unchanged and slightly reduces by $10 \%$ for $L_{d}=1 \mathrm{~nm}$ 


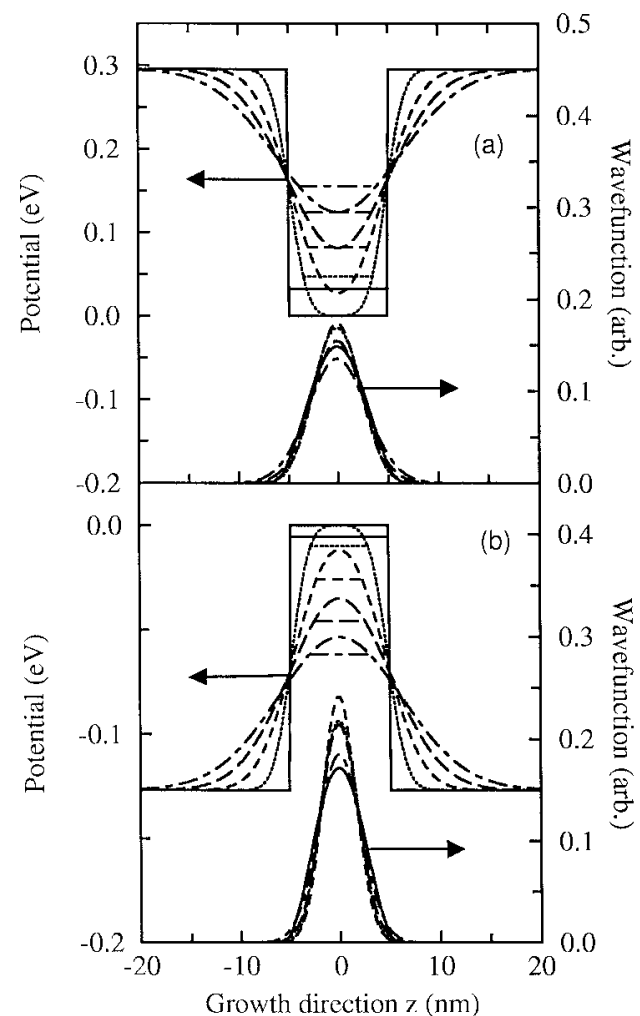

Fig. 1. The potential profile, wavefunction of the first eigenstate, and the states' well width of a $100-\AA-100-\AA \mathrm{Al}_{0.3} \mathrm{Ga}_{0.7} \mathrm{As}-\mathrm{GaAs} \mathrm{DFQW}$. (a) Conduction band. (b) Valence band. $L_{d}=0$ (solid line), $L_{d}=1 \mathrm{~nm}$ (dotted line), $L_{d}=2 \mathrm{~nm}$ (dashed line), $L_{d}=3 \mathrm{~nm}$ (long dashed line), and $L_{d}=$ $4 \mathrm{~nm}$ (dotted-dashed line).

and for $L_{d}=2 \mathrm{~nm}$, as shown in Fig. 1. These wavefunctions of both $\mathrm{C} 1$ and $\mathrm{HH} 1$ are therefore stronger confined with a higher wavefunction peak in the DFQW as compared to that of the as-grown structure $\left(L_{d}=0\right)$. As the interdiffusion proceeds further ( $\left.3 \mathrm{~nm} \leq L_{d} \leq 4 \mathrm{~nm}\right)$, the well depth becomes much lower and the wavefunctions of $\mathrm{C} 1$ and $\mathrm{HH} 1$ broaden out with their peaks reduced back to (or lower than) the as-grown case. The confinement only improves in the initial stages of interdiffusion $\left(L_{d} \leq 2 \mathrm{~nm}\right)$.

When the DFQW is tilted by an applied electric field, an interesting feature can be obtained, as shown in Fig. 2. For the case of $L_{d} \leq 2 \mathrm{~nm}$, the shift of wavefunctions weakens as compared to that of as-grown structure because of the enhanced confinement, while the wavefunction of the extensive interdiffused QW ( $\left.L_{d}=4 \mathrm{~nm}\right)$ shifts substantially and partly tunnel out. Consequently, the Stark shift of wavefunction can be tailored by using the QW interdiffusion.

The increase of $x$ from 0.2 to 0.4 (i.e., the increase of the QW barrier potential) improves the confinement in the asgrown QW. Its effect on the interdiffusion is best illustrated by the DFQW's with $L_{d}=2 \mathrm{~nm}$ under an applied field of $60 \mathrm{kV} / \mathrm{cm}$, as shown in Fig. 3(a)-(c). From these figures, the entire HH1 wavefunctions of the tilted DFQW move toward the centroid of the well as compared to the $\mathrm{HH} 1$ wavefunctions of the as-grown structure. Its peak amplitude, although slightly less than the as-grown ones, also increases when $x$ increases from 0.2 to 0.4 . This is contributed by the deepening of the

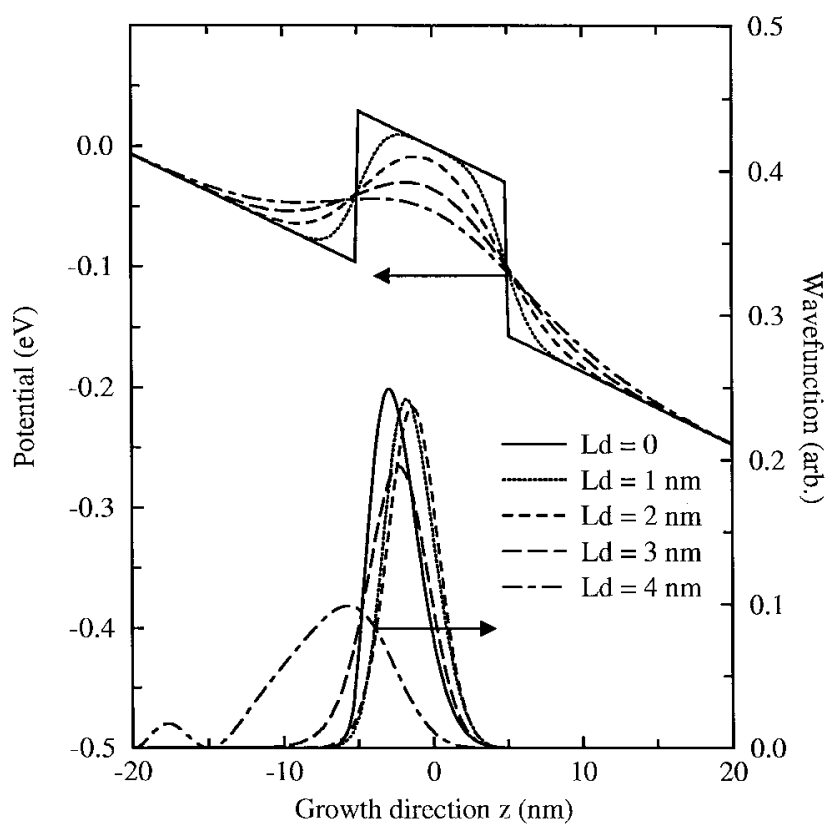

Fig. 2. $\mathrm{HH}$ potential profile and wavefunction of first $\mathrm{HH}$ eigenstate of $100-\AA-100-\AA \mathrm{Al}_{0.3} \mathrm{Ga}_{0.7}$ As-GaAs DFQW's with $F=60 \mathrm{kV} / \mathrm{cm} . L_{d}=$ 0 (solid line), $L_{d}=1 \mathrm{~nm}$ (dotted line), $L_{d}=2 \mathrm{~nm}$ (dashed line), $L_{d}=3$ $\mathrm{nm}$ (long dashed line), and $L_{d}=4 \mathrm{~nm}$ (dotted-dashed line).

potential depth and narrowing of the effective width due to a different magnitude of interdiffusion to the increasing $x$ content. For the $\mathrm{HH}$ valence band, the potential depth increases from 0.085 to $0.155 \mathrm{eV}$ and the effective width reduces from 5.54 to $4.66 \mathrm{~nm}$, as $x$ increases. This means that the biased first eigenstates are better confined with increasing $x$ content in the DFQW's.

More importantly, the biased eigenstates stay closer to the center of the DFQW as $x$ content increases. This is in contrary to the as-grown QW case in general. The consequence of this is a much improved overlapping between the electron and hole states under bias and will give rise to better modulation performance because we operate at biased exciton peak.

A similar feature of the HH1 wavefunction can also be found in $L_{d}=2 \mathrm{~nm}$ as the DFQW as-grown well width increases from $L_{z}=8 \mathrm{~nm}$ to $L_{z}=12 \mathrm{~nm}$, as shown in Fig. 4 . The wavefunctions stay closer to the central of QW as $L_{z}$ increases, although the amplitude of the $\mathrm{HH} 1$ wavefunction of the DFQW reduces in the widening of the as-grown well width. It is interesting to note that interdiffusion offers a special modification to the confinement of the biased first eigenstates. Generally, when well width increases, the confinement and their overlapping reduces. However, as shown in Fig. 4, the confinement of the HH1 wavefunction in the tilted DFQW's illustrates that it enhances the centralization of the eigenstates with the increase of the as-grown well width. This enhancement in the DFQW can be explained by the increase of the potential depth, which increases from 0.103 to $0.121 \mathrm{eV}$ as $L_{z}$ increases from 8 to $12 \mathrm{~nm}$. The effective well width also increases from 5.68 to $6.65 \mathrm{~nm}$ at the same time. This implies that the potential depth is the dominant factor contributed to the centralization. Although the effects of the two initial conditions, i.e., the value of $x$ and the as-grown well-width, on 


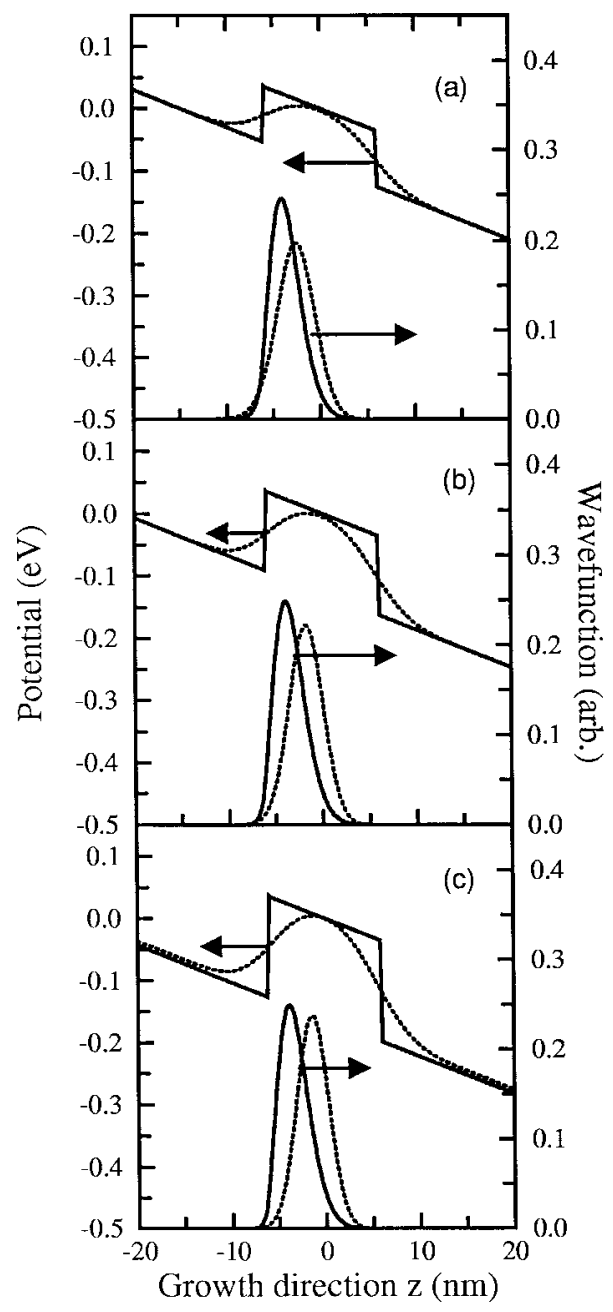

Fig. 3. As-grown $\mathrm{Al}$ concentration effect: $\mathrm{HH}$ potential profile and wavefunction of first $\mathrm{HH}$ eigenstate of the $\mathrm{Al}_{x} \mathrm{Ga}_{1-x} \mathrm{As}-\mathrm{GaAs} \mathrm{DFQW}$ with $L_{z}=$ $12 \mathrm{~nm}$ and $F=90 \mathrm{kV} / \mathrm{cm}$ The solid line represents $L_{d}=0$ (rectangular QW), and the dotted line represents $L_{d}=2 \mathrm{~nm}$ for the case of (a) $x=0.2$, (b) $x=0.3$, and (c) $x=0.4$.

the $\mathrm{C} 1$ confinements and its wavefunctions of DFQW's have not been discussed here, the obtained results show that their effects on $\mathrm{C} 1$ are the same as those on $\mathrm{HH} 1$. As a consequence, interdiffusion can recover the poor performance in a widewell-width QW which is used as an active region to produce a large Stark shift and a low $\alpha_{\text {loss }}$ in the EA modulator [20].

\section{B. Interdiffusion Contributions to Modulation Properties}

In analyzing the EA modulation properties of DFQW's, the operation wavelength $\lambda_{\mathrm{op}}$ is set at the wavelength of the biased 1S HH exciton absorption peak. DFQW's under different strengths of applied electric field are being investigated. The use of QW interdiffusion to adjust $\lambda_{\mathrm{op}}$ is also investigated here; this tunability is defined as the difference of $\lambda_{\text {op }}$ of a DFQW to its corresponding as-grown QW. The OFFstate and ON-state absorption coefficients are defined here as the absorption coefficients of the biased $1 \mathrm{~S} \mathrm{HH}$ exciton absorption peak and of the unbiased rising absorption edge at the wavelength of the biased exciton peak, respectively. $\alpha_{\text {loss }}$ is considered as the OFF-state absorption coefficient.

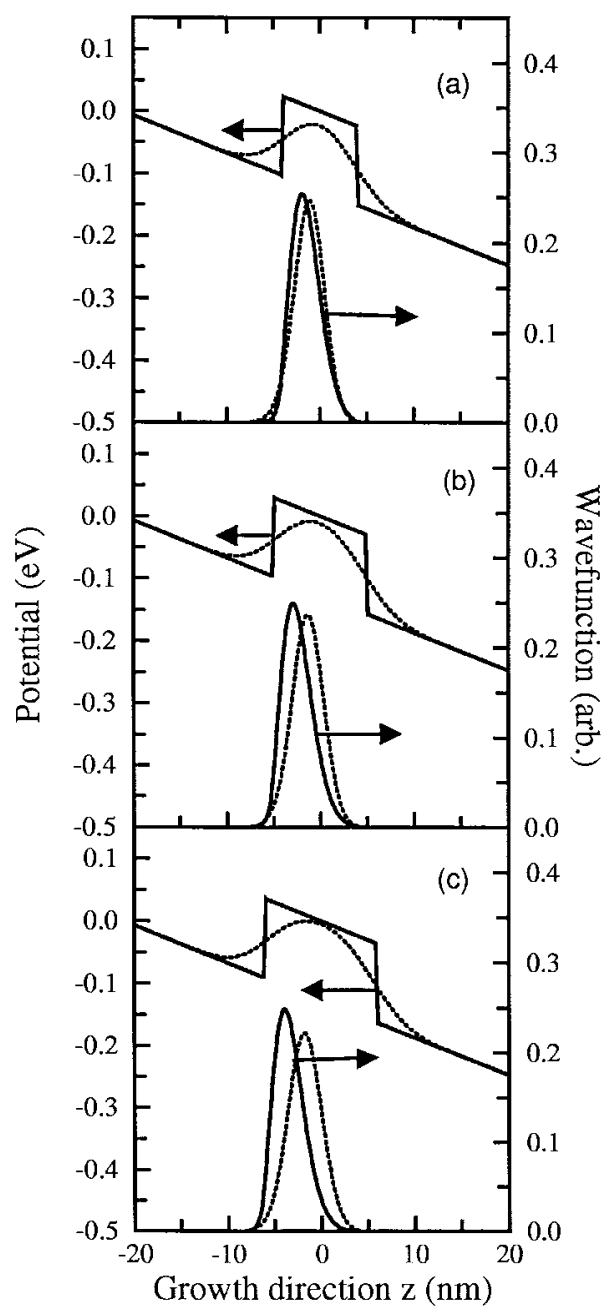

Fig. 4. As-grown well-width effect: the HH potential profile and wavefunction of the first $\mathrm{HH}$ eigenstate of the $\mathrm{Al}_{0.3} \mathrm{Ga}_{0.7}$ As-GaAs DFQW with $F=$ $90 \mathrm{kV} / \mathrm{cm}$. Solid line represents $L_{d}=0$ (rectangular QW), and dotted line represents $L_{d}=2 \mathrm{~nm}$. (a) $L_{z}=8 \mathrm{~nm}$. (b) $L_{z}=10 \mathrm{~nm}$. (c) $L_{z}=12 \mathrm{~nm}$.

1) Fixed As-Grown $Q W$ Conditions: The absorption spectra of the typical QW structure under different extents of interdiffusion $\left(0 \leq L_{d} \leq 4 \mathrm{~nm}\right)$ are shown in Fig. 5(a). The DFQW 1S HH exciton absorption peaks remain fairly constant with interdiffusion. However, with increasing interdiffusion, the $1 \mathrm{~S} \mathrm{HH}$ exciton peak wavelength blue shifts at a different rate. Under an applied electric field and thus producing the quantum-confined Stark effect, the exciton absorption peak of the DFQW's red shift and its amplitude reduce, such as for the care of DFQW with $L_{d}=2 \mathrm{~nm}$ shown in Fig. 5(b).

$\Delta \alpha$, i.e., the difference between the ON- and OFF-states absorption at different applied fields of this DFQW, is extracted to generate the dashed line in Fig. 6(b). Similarly, $\Delta \alpha$ of other $L_{d}$ cases are extracted to form the other lines in Fig. 6(b). It should be noted that, due to an enhanced confinement in the cases of $L_{d}=1$ and $2 \mathrm{~nm}, \Delta \alpha$ at different applied fields from $F=50-130 \mathrm{kV} / \mathrm{cm}$, i.e., dotted and dashed lines of Fig. 6(b), respectively, are greater than that of the as-grown case (solid line). When interdiffusion increases to $L_{d} \geq 3 \mathrm{~nm}$, its $\Delta \alpha$ at different applied fields reduces. The $\Delta \alpha$ of $L_{d}=3 \mathrm{~nm}$ terminates at $F=110 \mathrm{kV} / \mathrm{cm}$ because their $1 \mathrm{~S} \mathrm{HH}$ exciton tunnels 

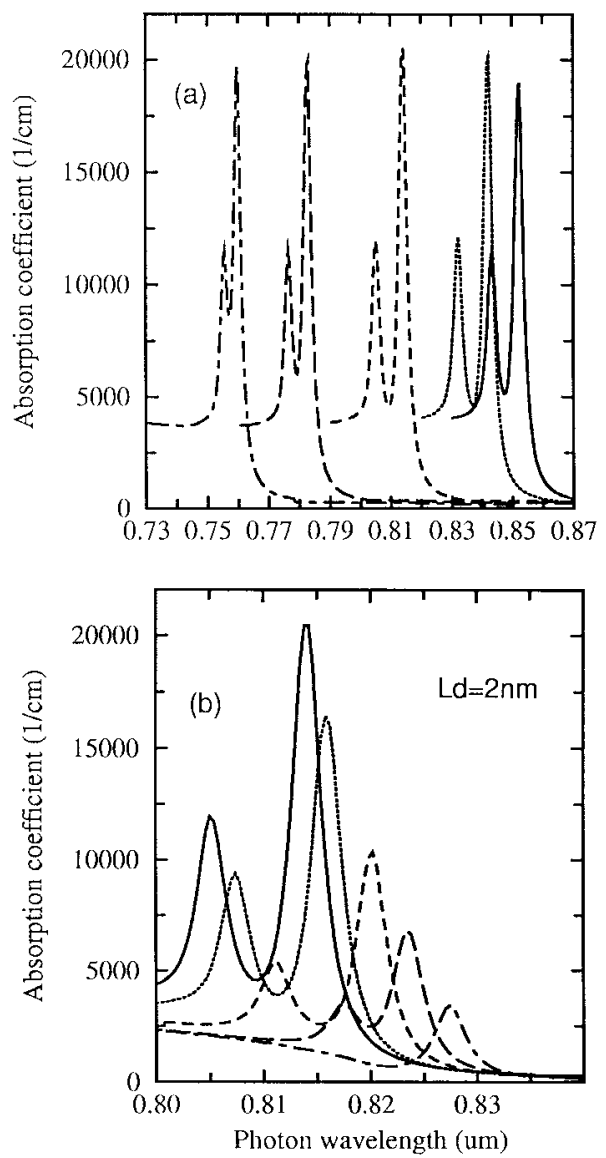

Fig. 5. The absorption coefficient of $\mathrm{Al}_{0.3} \mathrm{Ga}_{0.7} \mathrm{As}-\mathrm{GaAs} \mathrm{DFQW}$. (a) The absorptive spectra of the unbiased DFQW with $L_{d}$ from 0 to $4 \mathrm{~nm}$ stepped by $1 \mathrm{~nm} ; L_{d}=0$ (solid line), $L_{d}=1 \mathrm{~nm}$ (dotted line), $L_{d}=2 \mathrm{~nm}$ (dashed line), $L_{d}=3 \mathrm{~nm}$ (long dashed line), and $L_{d}=4 \mathrm{~nm}$ (dotted-dashed line). (b) The absorptive spectra of the biased DFQW with $L_{d}=2 \mathrm{~nm}, F=0$ (solid line), $F=50 \mathrm{kV} / \mathrm{cm}$ (dotted line), $F=90 \mathrm{kV} / \mathrm{cm}$ (dashed line), $F=$ $110 \mathrm{kV} / \mathrm{cm}$ (long dashed line), and $F=130 \mathrm{kV} / \mathrm{cm}$ (dotted-dashed line).

out. For even more extensive interdiffusion $\left(L_{d}=4 \mathrm{~nm}\right)$, it HH1 wavefunction is weakly bounded even under a small applied field of $F=60 \mathrm{kV} / \mathrm{cm}$, as can be seen in Fig. 2, and its $1 \mathrm{~S} \mathrm{HH}$ exciton tunnels out at $F \geq 90 \mathrm{kV} / \mathrm{cm}$. Its $\Delta \alpha$ can only shown up to $F=80 \mathrm{kV} / \mathrm{cm}$. For developing a large EA modulation, the use of DFQW, for example, with $L_{d}=1 \mathrm{~nm}$ at $F=110 \mathrm{kV} / \mathrm{cm}$, can produce an improved (twofold) $4.6-\mathrm{dB}$ CR per micrometer (propagation length) as compared to the as-grown $\mathrm{QW}$ of $2.3 \mathrm{~dB}$ at this applied field.

An important intrinsic parameter for the EA modulation is the quantum-confined Stark shift, because a large shift provides a cleaner and larger ON/OFF ratio. As listed in Table I (column 4), the Stark shift reduces with interdiffusion until $L_{d}=2 \mathrm{~nm}$ because of the enhanced confinement. When $L_{d}>2 \mathrm{~nm}$, the Stark shift increases due to the relaxation of confinement, as shown in Fig. 2. The Stark shift is also dominated by the valence band since the potential barrier of the valence band is shorter than that of the conduction band.

$\alpha_{\text {loss }}$ depends on the tail of rising unbiased absorption exciton edge and is inversely proportional to the Stark shift. The increase (although small) of unbiased absorption coefficient due to initial interdiffusion, as shown in Fig. 5(a), and the reduction of Stark shift, see Table I, make $\alpha_{\text {loss }}$ increase for $L_{d} \leq 2 \mathrm{~nm}$, as shown in Table II. However, $\alpha_{\text {loss }}$ reduces at $L_{d}=3 \mathrm{~nm}$ due to an increasing Stark shift. For more extensive interdiffusion, the $1 \mathrm{~S} \mathrm{HH}$ exciton tunnels out beyond $F=$ $90 \mathrm{kV} / \mathrm{cm}$, therefore, a slightly lower field $(F=80 \mathrm{~V} / \mathrm{cm})$ is used. Under this field strength, $\alpha_{\text {loss }}$ is only $\approx 580 \mathrm{~cm}^{-1}$ while the Stark shift is $24 \mathrm{meV}$. It should be noted that the $\alpha_{\text {loss }}$ considered here is purely the material absorption coefficient. In the case of a practical device structure, the optical confinement is usually $<1$ [21]. Take the optical confinement equal to 0.5 as an example, the $\alpha_{\text {loss }}$ of the extensive interdiffused QW ( $L_{d}=4 \mathrm{~nm}$ ) reduces to $290 \mathrm{~cm}^{-1}$.

Another important modulation parameter is the required bias (voltage) to produce the ON/OFF modulation. For the case of extensive interdiffusion, since the confinement becomes effectively weaker, the DFQW offers a higher tunneling rate [22]. Equivalently, a lower voltage is required [23] using the DFQW as compared to the as-grown QW. This higher switching rate combined with a low $\alpha_{\text {loss }}\left(580 \mathrm{~cm}^{-1}\right.$ at $F=$ $80 \mathrm{kV} / \mathrm{cm}$ ) can be very useful in high-speed EA modulators, while the lowered voltage feature can be used to satisfy the low bias requirement of switching arrays from a self-electrooptic effect device (SEED) [24].

An interesting feature of the DFQW is in its adjustability of $\lambda_{\mathrm{op}}$. A summary of $\lambda_{\mathrm{op}}$ working at $F=90 \mathrm{kV} / \mathrm{cm}$ as a function of $L_{d}$ (from 0 to $4 \mathrm{~nm}$ ), $x$ (from 0.2 to 0.4 ), and $L_{z}$ (from 8 to $12 \mathrm{~nm}$ ) are shown in Fig. 7. Due to the diffused potential profile moving upwards with increasing $L_{d}$, for the cases of $F \geq 0$, the energy of eigenstate in general increases with respect to that of the as-grown structure for cases of $F \geq 0$, and thus $\lambda_{\text {op }}$ blue shifts. For the typical case which is denoted by $(\cdots \cdots)$ in the figure, the $\lambda_{\mathrm{op}}$ blue shift from 1.442 to $1.565 \mathrm{eV}$ when $L_{d}$ increases from 0 to $4 \mathrm{~nm}$. This blue-shift property of $\lambda_{\mathrm{op}}$ is nonlinear in nature and that the tuning of $\lambda_{\mathrm{op}}$ is wider in the intermediate interdiffusion range $\left(1 \mathrm{~nm} \leq L_{d} \leq 2.5 \mathrm{~nm}\right)$ than those in the initial stage $\left(L_{d}<\right.$ $1 \mathrm{~nm})$ and final stage $\left(L_{d}>2.5 \mathrm{~nm}\right)$. In practice, the precise control of the operation wavelength by a fine adjustment of $L_{d}$ can be realized when the annealing temperature reduces to around $900{ }^{\circ} \mathrm{C}$ [25]. For instance, an annealing time of $10 \mathrm{~s}$ implies $L_{d}=\sqrt{\left[\left(2.9 \times 10^{-17} \mathrm{~cm}^{2} / \mathrm{s}\right)(10 \mathrm{~s})\right]}=1.7 \AA$. Therefore, the required $L_{d}$ for a targeted wavelength discussed here is experimentally achievable.

2) Effect of As-Grown Al Concentration Variation in Barrier: When $x$ increases from 0.2 to 0.4 and $L_{z}$ is fixed at $12 \mathrm{~nm}, \Delta \alpha$ under different applied fields and for all interdiffusion extents gradually rises up due to an enhancement of the quantum confinement, as shown by Fig. 6(c)-(e). The increasing DFQW $\Delta \alpha$ over its corresponding as-grown $\mathrm{QW}$ enhances from the case of $x=0.2$ to $x=0.4$. As a consequence, when $\mathrm{Al}$ concentration increases, a longer range of interdiffusion exists with an enhanced $\Delta \alpha$, as well as an increased magnitude of $\Delta \alpha$. However, it should be noted that, for all cases of $x, \Delta \alpha$ terminates at a lower applied electric field when $L_{d}$ increases, see Fig. 6(c)-(e). Generally, the maximum allowed applied field (voltage) of DFQW's increases when $x$ increases. 


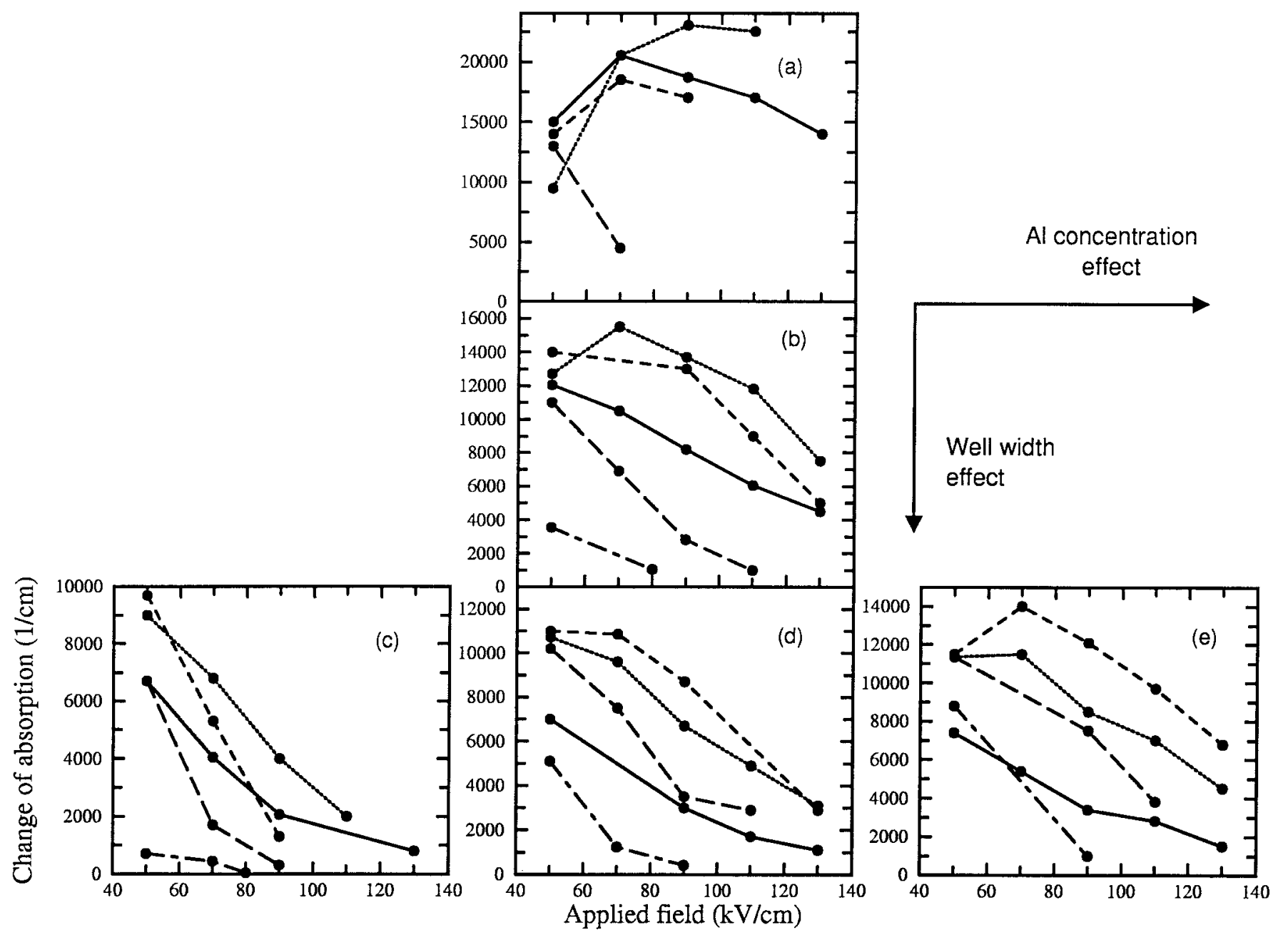

Fig. 6. The effects of $\mathrm{Al}$ concentration $x$ and as-grown well width $\left(L_{z}\right)$ on the absorption change of $\mathrm{Al}_{x} \mathrm{Ga}_{1-x} \mathrm{As}-\mathrm{GaAs} \mathrm{DFQW}$ 's: (a) $x=0.3$ and $L_{z}=8$ $\mathrm{nm}$, (b) $x=0.3$ and $L_{z}=10 \mathrm{~nm}$, (c) $x=0.2$ and $L_{z}=12 \mathrm{~nm}$, (d) $x=0.3$ and $L_{z}=12 \mathrm{~nm}$, and (e) $x=0.4$ and $L_{z}=12 \mathrm{~nm}$, in different $L_{d}$ from 0 to $4 \mathrm{~nm}$ stepped by $1 \mathrm{~nm}$. $L_{d}=0$ (solid line), $L_{d}=1 \mathrm{~nm}$ (dotted line), $L_{d}=2 \mathrm{~nm}$ (dashed line), $L_{d}=3 \mathrm{~nm}$ (long dashed line), and $L_{d}=4 \mathrm{~nm}$ (dotted-dashed line).

TABLE I

Stark Shift And the Normalized Shift, I.E., Stark $\operatorname{shift}(L d \neq 0) / \operatorname{Stark} \operatorname{shift}(L d=0)$ Which Is Denoted as $\mathrm{SS}_{L d \neq 0} / \mathrm{SS}_{L d=0}$, OF DFQW'S WITH $L_{d}=0-4 \mathrm{~nm}$ STEPPED By $1 \mathrm{NM}$ IN AN APPLIED Field $F=90 \mathrm{kV} / \mathrm{cm}$

\begin{tabular}{|c|c|c|c|c|c|c|c|c|c|c|}
\hline & \multicolumn{2}{|c|}{$\mathrm{x}=0.2, \mathrm{~L}_{\mathrm{z}}=12 \mathrm{~nm}$} & \multicolumn{2}{|c|}{$\mathrm{x}=0.3, \mathrm{~L}_{\mathrm{z}}=8 \mathrm{~nm}$} & \multicolumn{2}{|c|}{$\mathrm{x}=0.3, \mathrm{~L}_{\mathrm{z}}=10 \mathrm{~nm}$} & \multicolumn{2}{|c|}{$\mathrm{x}=0.3, \mathrm{~L}_{\mathrm{z}}=12 \mathrm{~nm}$} & \multicolumn{2}{|c|}{$\mathrm{x}=0.4, \mathrm{~L}_{\mathrm{z}}=12 \mathrm{~nm}$} \\
\hline $\begin{array}{l}\mathrm{L}_{\mathrm{d}} \\
(\mathrm{nm})\end{array}$ & $\begin{array}{l}\text { Shift } \\
(\mathrm{meV})\end{array}$ & $\begin{array}{l}\mathrm{SS}_{\mathrm{Ld} \times 1} \\
\mathrm{SS}_{\mathrm{Id} \|}\end{array}$ & $\begin{array}{l}\text { Shift } \\
(\mathrm{meV})\end{array}$ & $\begin{array}{l}S_{L S_{L \neq 0}} \\
S S_{L d=0}\end{array}$ & $\begin{array}{l}\begin{array}{l}\text { Shift } \\
(\mathrm{meV})\end{array}\end{array}$ & $\begin{array}{l}\mathrm{SS}_{\mathrm{Ld \neq 0}} \\
\mathrm{SS}_{\mathrm{Id}=0}\end{array}$ & \begin{tabular}{|l|} 
Shift \\
$(\mathrm{meV})$
\end{tabular} & $\begin{array}{l}\mathrm{SS}_{\mathrm{Ld}+d} \\
\mathrm{SS}_{\mathrm{Id}=0}\end{array}$ & \begin{tabular}{|l|} 
Shift \\
(meV)
\end{tabular} & $\begin{array}{l}\mathrm{SS}_{\mathrm{Ld} \neq 0} \\
\mathrm{SS}_{\mathrm{Ld}=0}\end{array}$ \\
\hline 0 & 32.9 & 1 & 11.1 & 1 & 20.2 & 1 & 30.4 & 1 & 28.1 & 1 \\
\hline 1 & 25.5 & 0.76 & 6.5 & 0.59 & 12.2 & 0.60 & $\mid 19.4$ & 0.64 & 17.0 & 0.60 \\
\hline 2 & 21.5 & 0.65 & 10.0 & 0.90 & 11.1 & 0.55 & 14.8 & 0.49 & 12.7 & 0.45 \\
\hline 3 & -- & -- & -- & -- & 18.2 & 0.90 & 15.3 & 0.50 & 15.9 & 0.57 \\
\hline 4 & -- & -- & -- & -- & -- & -- & -- & -- & 26.3 & 0.94 \\
\hline
\end{tabular}

TABLE II

Residence Loss of DFQW's that the Operation Wavelength is Selected at Bias Exciton Peak $(F=90 \mathrm{kV} / \mathrm{cm})$ and the Normal ON-State is at $F=0$

\begin{tabular}{l|lll|lll|lll}
\hline & \multicolumn{9}{|c|}{ absorption loss $\left(\mathrm{cm}^{-1}\right)$} \\
\hline $\mathrm{L}_{\mathrm{d}}$ & \multicolumn{3}{|c|}{$\mathrm{x}=0.2$} & \multicolumn{3}{c|}{$\mathrm{x}=0.3$} & \multicolumn{3}{c}{$\mathrm{x}=0.4$} \\
\cline { 2 - 10 }$(\mathrm{nm})$ & $\mathrm{L}_{\mathrm{z}}=8 \mathrm{~nm}$ & $\mathrm{~L}_{\mathrm{z}}=10 \mathrm{~nm}$ & $\mathrm{~L}_{\mathrm{z}}=12 \mathrm{~nm}$ & $\mathrm{~L}_{\mathrm{z}}=8 \mathrm{~nm}$ & $\mathrm{~L}_{\mathrm{z}}=10 \mathrm{~nm}$ & $\mathrm{~L}_{\mathrm{z}}=12 \mathrm{~nm}$ & $\mathrm{~L}_{\mathrm{z}}=8 \mathrm{~nm}$ & $\mathrm{~L}_{\mathrm{z}}=10 \mathrm{~nm}$ & $\mathrm{~L}_{\mathrm{z}}=12 \mathrm{~nm}$ \\
\hline 0 & 2457 & 990 & 606 & 3161 & 1169 & 657 & 3695 & 1278 & 730 \\
1 & 3630 & 1487 & 715 & 6653 & 2354 & 1000 & 10228 & 3171 & 1230 \\
2 & 2500 & 1275 & 892 & 3787 & 2558 & 1464 & 6586 & 4048 & 2243 \\
3 & -- & -- & -- & -- & 1250 & 1100 & 2065 & 1820 & 1483 \\
4 & -- & -- & -- & -- & -- & -- & -- & -- & 779 \\
\hline
\end{tabular}




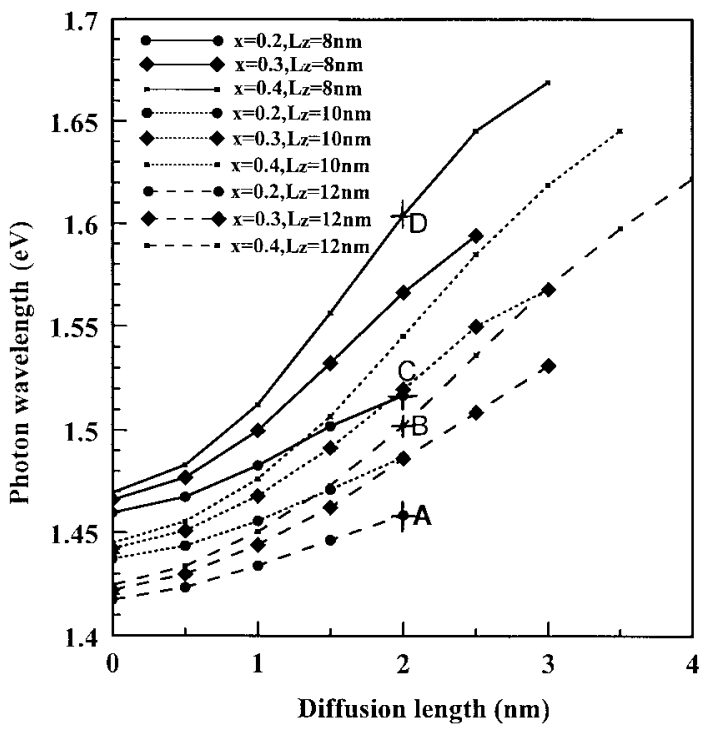

Fig. 7. The operation wavelengths of AlGaAs-GaAs DFQW in different $L_{d}$ from 0 to $4 \mathrm{~nm}$ stepped by $1 \mathrm{~nm}$. The operation wavelengths are at the $1 \mathrm{~S}$ $\mathrm{HH}$ exciton peak biased by applied field $F=90 \mathrm{kV} / \mathrm{cm}$. The solid, dotted, and dashed lines are the cases $L_{z}=8,10$, and $12 \mathrm{~nm}$, respectively. For a fixed diffusion, i.e., $L_{d}=2 \mathrm{~nm}$, the points $\mathrm{A}$ and $\mathrm{C}$ represent the wavelength at $L_{Z}=12$ and $8 \mathrm{~nm}$, respectively, for $x=0.2$. Likewise, the points B and D correspond to the cases for $x=0.4$.

The variation of the Stark shift due to the increase of $\mathrm{Al}$ concentration is better illustrated by its normalized value, i.e., Stark $\operatorname{shift}(L d \neq 0) /$ Stark $\operatorname{shift}(L d=0)$. The normalized Stark shift of the DFQW's reduces, see Table I (columns 2, 4, and 6), and thus $\alpha_{\text {loss }}$ of the DFQW's increases, see Table II, when $x$ increases. Adjustability of $\lambda_{\text {op }}$ of DFQW's also widens with the increase of $x$, as shown in Fig. 7. By comparing the adjustable range of $\lambda_{\mathrm{op}}$ of the DFQW (from $L_{d}=0$ to $L_{d}=$ $2 \mathrm{~nm}$ ), when $x$ increases through $0.2,0.3$, and then 0.4 , it increases from 35.4 to $64.3 \mathrm{meV}$, and $78.1 \mathrm{meV}$, respectively.

3) Effect of As-Grown Well Width Variation: The effect of as-grown well width on $\Delta \alpha$ of DFQW's are summarized in Fig. 6(a), (b), and (d). It is found that for almost all the interdiffusion cases and $L_{d}=0$ (the as-grown structure), $\Delta \alpha$ on the whole reduces with increasing $L_{z}$. However, the range of the interdiffusion extent with an enhanced the $\Delta \alpha$ (over that of $L_{d}=0$ case) widens when the as-grown well width increases. This is because the confinement of QW with wider as-grown well width improves with interdiffusion. In addition, interdiffusion can produce a recovery to the reduction of $\Delta \alpha$ due to increasing well width. For instance, when $L_{z}$ of an as-grown $\mathrm{QW}$ increases from 8 to $10 \mathrm{~nm}$, its $\Delta \alpha$ at $F=$ $110 \mathrm{kV} / \mathrm{cm}$ reduces from 17000 to $6,000 \mathrm{~cm}^{-1}$, however, the $\Delta \alpha$ of the later QW can be improved to $12000 \mathrm{~cm}^{-1}$ by interdiffusion to $L_{d}=1 \mathrm{~nm}$. Consequently, there are two benefits in using interdiffusion in a wide-well-width QW structure: 1) the range of an enhanced $\Delta \alpha$ increases when $L_{z}$ increases and 2) interdiffusion can, to a certain extent, compensate the degradation caused by a widened well width on $\Delta \alpha$.

As $L_{z}$ increases, the amount of Stark shift of all the interdiffusion cases increases, as shown in Table I (the middle three columns). The $\alpha_{\text {loss }}$ reduces as shown in Table II, and
TABLE III

NoRmaLIZED $\Delta \alpha$, I.E., $\Delta \alpha_{L d \neq 0} / \Delta \alpha_{L d=0}$, OF THE DFQW with RESPECT TO the Corresponding Rectangular QW'S For DifFerent $L_{d}$ CASES (From 1 to 4 NM STEPPED By $1 \mathrm{~nm}$ ) UNDER APPLIEd Field $F=90 \mathrm{kV} / \mathrm{cm}$

\begin{tabular}{l|llll} 
& \multicolumn{5}{|c}{$\left(\Delta \alpha_{\mathrm{Ld} \neq 0}\right) /\left(\Delta \alpha_{\mathrm{Ld} \neq 0}\right)$} \\
\hline $\mathrm{x}=0.2$ & $\mathrm{~L}_{\mathrm{d}}=1 \mathrm{~nm}$ & $\mathrm{~L}_{\mathrm{d}}=2 \mathrm{~nm}$ & $\mathrm{~L}_{\mathrm{d}}=3 \mathrm{~nm}$ & $\mathrm{~L}_{\mathrm{d}}=4 \mathrm{~nm}$ \\
\hline $\mathrm{L}_{\mathrm{z}}=8 \mathrm{~nm}$ & 1.17 & 0.53 & - & -- \\
$\mathrm{L}_{\mathrm{z}}=10 \mathrm{~nm}$ & 1.37 & 0.73 & -- & -- \\
$\mathrm{L}_{\mathrm{z}}=12 \mathrm{~nm}$ & 1.86 & 0.65 & -- & -- \\
\hline $\mathrm{x}=0.3$ & & & & \\
$\mathrm{~L}_{\mathrm{z}}=8 \mathrm{~nm}$ & 1.23 & 0.96 & - & -- \\
$\mathrm{L}_{\mathrm{z}}=10 \mathrm{~nm}$ & 1.76 & 1.70 & 0.41 & -- \\
$\mathrm{L}_{\mathrm{z}}=12 \mathrm{~nm}$ & 2.22 & 2.91 & 1.16 & - \\
\hline $\mathrm{x}=0.4$ & & & & \\
$\mathrm{Lz}=8 \mathrm{~nm}$ & 1.19 & 1.15 & 0.09 & - \\
$\mathrm{Lz}=10 \mathrm{~nm}$ & 1.80 & 1.90 & 0.91 & -- \\
$\mathrm{Lz}=12 \mathrm{~nm}$ & 2.46 & 3.58 & 2.18 & 0.34 \\
\hline
\end{tabular}

the adjustability of $\lambda_{\mathrm{op}}$ decreases as the as-grown well width widens. On the other hand, since a narrower as-grown width QW will have a more rapid rate of reduction in the diffused potential depth (thus weakening the confinement), the applied voltage for a required level of ON/OFF in the extensively interdiffused QW is expected to be lowered by reducing the well width, see Fig. 6(d) to Fig. 6(b) to Fig. 6(a).

\section{COMBined EFFects of the Initial CONDITIONS}

a) In general, an increase of both $\mathrm{Al}$ concentration and well width widens the interdiffusion range with an improved $\Delta \alpha$, as shown in Fig. 6. An illustrative example is shown in Table III which lists the normalized $\Delta \alpha$ $\left(\Delta \alpha_{L d \neq 0} / \Delta \alpha_{L d=0}\right)$ of DFQW's under bias $F=90$ $\mathrm{kV} / \mathrm{cm}$. By comparing the enhanced $\Delta \alpha$ caused by increasing $\mathrm{Al}$ concentration with that due to the increase of well width, it can be observed that the former one is more obvious and dominating. Take DFQW with $L_{d}=$ $1 \mathrm{~nm}$ as an example and use the ratio of the different normalized $\Delta \alpha$ cases for comparison. The increment of $x$ from 0.2 to 0.4 provides an enhancement to the effect of widening $L_{z}$ on the DFQW from 1.86/1.17 $=1.59$ to $2.46 / 1.19=2.07$. The widening of $L_{z}$ from 8 to $12 \mathrm{~nm}$, however, brings a weaker enhancement to the effect of increasing $x$ on the DFQW from 1.19/1.17 $=$ 1.05 to $2.46 / 1.86=1.32$. This means that the effect of interdiffusion on $\Delta \alpha$ is intensified by an increasing $\mathrm{Al}$ concentration of the QW in the study ranges considered here for the two initial conditions. This is in agreement with the analysis of Section III-A that the dominant factor of an enhanced confinement and thus an improved $\Delta \alpha$ [26] is the diffused potential depth rather than the diffused well width.

b) The variations of $\alpha_{\text {loss }}$ and Stark shift in the DFQW's, caused by a change of the $\mathrm{Al}$ concentration and asgrown well width, are inversely related to each other; the former one increases when $\mathrm{Al}$ concentration increases and $L_{z}$ decreases, and vice verse for the later one. Of the two effects, the variation of $\alpha_{\text {loss }}$ and Stark shift strongly depends on the $\mathrm{Al}$ concentration and as-grown well width, respectively. This can be seen by comparing the ratio of the normalized values as used in a). 
c) An enlargement of the $\mathrm{Al}$ concentration and a reduction of $L_{z}$ can widen the adjustable range of $\lambda_{\mathrm{op}}$. With the initial condition of $x$ increased from 0.2 to 0.4 , the variation of $\lambda_{\text {op }}$ of a DFQW (at $L_{d}=2 \mathrm{~nm}$ and $F=$ $90 \mathrm{kV} / \mathrm{cm}$ ) between $L_{z}=8$ and $12 \mathrm{~nm}$ will be modified from a bandwidth of $\mathrm{AC}$ to $\mathrm{BD}$, see Fig. 7. Their relative ratio, i.e., $\mathrm{BD} / \mathrm{AC}$, is 1.75 which shows the effect of increment in $x$. With the initial condition of $L_{z}$ reduced from 12 to $8 \mathrm{~nm}$, the modification of $\lambda_{\mathrm{op}}$ of the DFQW due to an increment in $x$ from 0.2 to 0.4 will change from the width $\mathrm{AB}$ to $\mathrm{CD}$. The influence of reduction in $L_{z}$ produces a relative ratio (i.e., $\mathrm{CD} / \mathrm{AB}$ ) of 1.99 . Therefore, it can be concluded that the adjustability of $\lambda_{\text {op }}$ through interdiffusion is more sensitively dependent on the as-grown well width.

d) One of the interesting features of the DFQW is a lowered required voltage swing when the interdiffusion is moderately extensive (the relevant value of $L_{d}$ depends on the Al concentration and $L_{z}$ ). The maximum allowed applied voltage increases with the increase of both $x$ and $L_{z}$.

\section{AdVANTAGES OF EMPLOYING DFQW'S AS THE ACTIVE CAVITY MATERIAL}

Based on the four features discussed in Section IV, for general applications, the $\mathrm{Al}_{x} \mathrm{Ga}_{1-x} \mathrm{As}-\mathrm{GaAs}$ with $x$ between 0.3 and 0.4 and $L_{z}$ between 10 and $12 \mathrm{~nm}$ should be employed to develop DFQW EA modulation devices for general applications. Within these ranges, the magnitudes of $\Delta \alpha$, the extension of $L_{d}$ with improved $\Delta \alpha$, and the adjustability of $\lambda_{\mathrm{op}}$ are at their best.

A wide-well-width rectangular QW to be used as the activeregion material can provide a large Stark shift and a low $\alpha_{\text {loss }}$ in the EA modulator [20]. With interdiffusion, the magnitude of $\Delta \alpha$ can be further improved in this wide-well-width QW system. The range of interdiffusion with an enhanced $\Delta \alpha$ widens with an increasing $L_{z}$. On the other hand, low-Al concentration in rectangular QW's are attractive instruments for developing SEED devices [20] since it provides a fast tunneling rate. However, these QW's suffer from low $\Delta \alpha$. Interdiffusion can then be a remedy to restore the $\Delta \alpha$, as demonstrated in Fig. 7.

The only drawbacks of the DFQW for the above two devices seem to be a narrow Stark shift and a large $\alpha_{\text {loss }}$. However, for an accurate comparison, a DFQW should be compared with an "equivalent" rectangular QW [27] having the same amount of Stark shift. In this case, the $\alpha_{\text {loss }}$ of DFQW is found to be less. In order to develop an equivalent $\mathrm{QW}$ with the same $\Delta \alpha$ as that of the DFQW, a narrower well width or a deeper potential depth is required. This will reduce the Stark shift and the tunneling sensitivity [20], thus increasing the loss as compared to the DFQW. On the other hand, in order to provide a similar Stark shift to the DFQW structures, wider well width and shallower well depth in the equivalent QW are required. In this case, the $\Delta \alpha$ of the equivalent $\mathrm{QW}$ reduces. This means that an equivalent QW cannot attain both the $\Delta \alpha$ and Stark shift (and thus residual loss) of a DFQW at the same time. As a whole, the improvement of DFQW in terms of confinement and tunneling sensitivity cannot be replaced by a single rectangular QW. This is also one of the advanced features in using a DFQW for the EA modulator.

The required bias of the modulator can also be reduced by introducing an extensively interdiffused QW structure, thus found application in a high-speed modulator. DFQW can provide an adjustability of $\lambda_{\mathrm{op}}$, therefore, we only require a single substrate QW structure with a selective interdiffusion process to develop EA modulators which operate at different $\lambda_{\text {op. }}$. A multiple-bandgap integrated structure can be realized for multi-colors or wide-bandwidth applications. The yield of fabrication can also be improved by using interdiffusion as a post-growth wavelength correction technique.

\section{CONCLUSION}

We have theoretically analyzed the modulation properties of an $\mathrm{Al}_{x} \mathrm{Ga}_{1-x} \mathrm{As}-\mathrm{GaAs}$ DFQW in terms of the initial conditions prior to interdiffusion, i.e., the effects of $\mathrm{Al}$ concentration $(x=0.2,0.3$, and 0.4$)$, and well width $\left(L_{z}=8,10\right.$, and 12 $\mathrm{nm})$. The results show that: 1) the interdiffusion range with improved $\Delta \alpha$ increases with increasing both $x$ and $L_{z} ; 2$ ) $\alpha_{\text {loss }}$ increases with increasing $x$ and reducing $L_{z}$ especially in the middle range of interdiffusion where the increment is more serious; 3) Stark shift first reduces in a less extensive DFQW and then increases with further interdiffusion, and the shift increases with the reduction of $x$ and the increment of $L_{z}$; 4) the required voltage for ON/OFF operation reduces in larger interdiffusion extent, however extensive interdiffusion is required for low-voltage applications when both $x$ and $L_{z}$ increases; 5) interdiffusion can provide an adjustable $\lambda_{\text {op }}$ range, which increases with increasing $x$ and decreasing $L_{z}$; and 6) $\mathrm{Al}$ concentration is the dominant factor contributing to $\Delta \alpha$ and $\alpha_{\text {loss }}$ while Stark shift and adjustability of $\lambda_{\mathrm{op}}$ are dominated by as-grown well width.

In general applications, the $\mathrm{Al}_{x} \mathrm{Ga}_{1-x} \mathrm{As}-\mathrm{GaAs}$ DFQW with $x$ between 0.3 and 0.4 and $L_{z}$ between 10 and $12 \mathrm{~nm}$ should be employed to develop EA modulators. Interdiffusion can also be applied to the wide-width rectangular QW system, which is commonly used in high-speed modulators, to produce a large $\Delta \alpha$ and a wide range of $L_{d}$ with enhanced $\Delta \alpha$.

For low $\mathrm{Al}$ concentration and wide $L_{z} \mathrm{QW}$ materials which are used in high-speed modulators, the DFQW's developed from these as-grown materials can provide an even larger $\Delta \alpha$. The Stark shift enlarges in the DFQW's, however, residual loss increases although it is comparatively small as compared with the case of rectangular QW with the same $\Delta \alpha$. The improved quantum confinement and a higher tunneling rate achieved in the DFQW's cannot be fulfilled by a single rectangular QW structure simultaneously. The extensively interdiffused QW reduces the required bias or increases the tunneling rate for EA modulation. Therefore, the DFQW material structure is potentially attractive for developing high-speed modulators. The adjustability of $\lambda_{\mathrm{op}}$ in the DFQW enables successful development of a single substrate QW structure to operate at different photon wavelengths by a selective-area interdiffusion process on the wafer. The yield of device fabrication can 
also be improved by using interdiffusion as a post-growth wavelength correction technique. All these attractive features will undoubtedly make the DFQW's a competitive material for the development of EA modulators and their integration.

\section{REFERENCES}

[1] T. H. Wood, C. A. Burrus, D. A. B. Miller, D. S. Chemla, T. C. Damen, A. C. Gossard, and W. Wiegmann, "High-speed optical modulation with GaAs/AlGaAs quantum wells in a p-i-n diode structure," Appl. Phys. Lett., vol. 44, pp. 16-18, 1984.

[2] T. Ido, H. Sano, M. Sano, M. Suzuki, S. Tanaka, and H. Inoue, "High-speed MQW electroabsorption optical modulators integrated with low-loss waveguides," IEEE Photon. Technol. Lett., vol. 7, pp. 170-172, 1995.

[3] J. D. Ralston, W. J. Schaff, D. P. Bour, and L. F. Eastman, "Room-temperature exciton electroabsorption in partially intermixed GaAs/AlGaAs quantum well waveguides," Appl. Phys. Lett., vol. 54, pp. $534-536,1988$

[4] M. Ghisoni, G. Parry, M. Pate, G. Hill, and J. Roberts, "Post growth fabrication of $\mathrm{GaAs} / \mathrm{AlGaAs}$ reflection modulators via impurity free disordering," Jpn. J. Appl. Phys., vol. 30, pp. L1018-L1020, 1991.

[5] W. C. H. Choy and E. H. Li, "The applications of interdiffused quantum well in normally-on electro-absorptive Fabry-Perot reflection modulator," IEEE J. Quantum Electron., vol. 33, pp. 382-393, 1997.

[6] J. E. Zucker, K. L. Jones, B. Tell, K. Brown-Goebeler, C. H. Joyner B. L. Miller, and M. G. Young, "InGaAsP/InP Quantum well buried herterostructure waveguides produced by ion implantation," Electron. Lett., vol. 28, pp. 853-855, 1992.

[7] I. Gontijo, T. Krauss, R. M. De La Rue, J. S. Roberts, and J. H. Marsh, "Very low loss extended cavity GaAs/AlGaAs lasers made by impurity-free vacancy diffusion," Electron. Lett., vol. 30, pp. 145-146, 1994.

[8] J. J. He, Y. Feng, E. S. Koteles, P. J. Poole, M. Davis, M. Dion, R. Goldberg, I. Mitchell, and S. Charbonneau, "Bandgap shifted InGaAsP/InP quantum well waveguides using $\mathrm{MeV}$ ion implantation," Electron. Lett., vol. 24, pp. 2094-2095, 1995.

[9] A. MiKee, C. J. Mchlean, G. Luulo, A. C. Catrina, R. M. De La Rue, J. H. March, and C. C. Button, "Monolithic integration in InGaAs-InGaAsP multiple-quantum-well structures," IEEE J. Quantum Electron., vol. 33, pp. 45- 54, 1997.

[10] T. E. Scholesinger and T. Kuech, "Determination of the interdiffusion of $\mathrm{Al}$ and $\mathrm{Ga}$ in undoped $(\mathrm{Al}, \mathrm{Ga}) \mathrm{As} / \mathrm{GaAs}$ quantum wells," Appl. Phys. Lett., vol. 49, pp. 519-521, 1986.

[11] W. Bloss, "Electronic field dependence of quantum-well eigenstates," $J$. Appl. Phys., vol. 65, pp. 4789-4794, 1989.

[12] E. H. Li, B. L. Weiss, and K. S. Chan, "Effect of interdiffusion on the subbands in an AlGaAs/GaAs single quantum well structure," Phys. Rev. B., vol. 46, pp. 15180-15191, 1992.

[13] T. F. Jiang, "An alternative approach of exciton binding energy in a $\mathrm{Al}_{x} \mathrm{Ga}_{1-x} \mathrm{As} / \mathrm{GaAs}$ quantum well," Solid State Commun.., vol. 50, pp. 589-593, 1984

[14] S. Adachi, "Optical properties of AlGaAs alloys," Phys. Rev. B, vol. 38, pp. 12345-12352, 1988.
[15] W. C. H. Choy, E. H. Li, and J. Micallef, "The polarization insensitive electro-absorptive and -refractive modulation by utilizing InGaAsP/InP interdiffused quantum well," IEEE J. Quantum Electron., vol. 33, pp. 1316-1322, 1997.

[16] J. Singh, S. Hong, P. K. Bhatacharya, R. Sahai, C. Lastufka, and H. R. Sobel, "System requirement and feasibility studies for optical modulators based on GaAs/AlGaAs multiquantum well structures for optical-processing," J. Lightwave Technol., vol. 6, pp. 818-831, 1988.

[17] T. Hayakawa, K. Takahashi, M. Kondo, T. Suyama, S. Yamamoto, and T. Hijikata, "Enhancement in optical transitions in (111)—oriented GaAs-AlGaAs quantum well structures," Phys. Rev. Lett., vol. 60, pp. 349-352, 1988 .

[18] M. Ghisoni, P. J. Stevens, G. Parry, and J. S. Roberts, "Post-growth tailoring of the optical properties of GaAs/AlGaAs quantum well structures," Opt. Quantum Electron., vol. 23, pp. S915-S924, 1991.

[19] E. H. Li and W. C. H. Choy, "Electro-absorption properties of Interdiffused InGaAsP/InP quantum wells," J. Appl. Phys., vol. 82, no. 8, pp. 3861-3869, Oct. 1997.

[20] A. M. Fox, D. A. B. Miller, G. Livescu, J. E. Cuningham, and W. Y. Jan, "Quantum well carrier sweep out: relation to electroabsorption and exciton saturation," IEEE J. Quantum Electron., vol. 27, pp. 2281-2295, 1991.

[21] T. C. Hasenberg, S. D. Kothler, D. Yap, A. Kost, and E. M. Garmine, "All-binary InAs/GaAs, optical waveguide phase modulator at 1.06 $\mu \mathrm{m}, "$ IEEE Photon Technol. Lett., vol. 6, pp. 1210-1212, 1994.

[22] Z. Yang, B. L. Weiss, and E. H. Li, "The eigenstates and tunneling lifetime in an interdiffused $\mathrm{AlGaAs} / \mathrm{GaAs}$ quantum well by the transfer matrix method," Superlattice Microstruct., vol. 17, no. 2, pp. 177-186, 1995.

[23] N. Debbar, S. Hong, J. Singh, P. Bhattacharya, and R. Sahai, "Coupled GaAs/AlGaAs-well electroabsorption modulators for low-electric-field optical modulation," J. Appl. Phys., vol. 65, pp. 383-385, 1989.

[24] D. A. B. Miller, "Optical bistablity in self-electro-optic effect devices with asymmetric quantum wells," Appl. Phys. Lett., vol. 54, pp. 202-204, 1989.

[25] P. J. Hughes, E. H. Li, and B. L. Weiss, "Thermal stability of AlGaAs/GaAs single QW structures using photo reflectance," J. Vac. Sci. Technol. B, vol. 13, pp. 2276- 2280, 1995.

[26] B. Pezeshki, S. M. Lord, T. B. Boykin, and J. S. Harris, Jr., "GaAs/AlAs quantum wells for electro-absorption modulators," Appl. Phys. Lett., vol. 60, pp. 2779-2781, 1992.

[27] E. H. Li, K. S. Chan, B. L. Weiss, and J. Micallef, "The quantum confined stark effect in an interdiffusion induced quantum well," Appl. Phys. Lett., vol. 63, no. 4, pp. 533-535, 1993.

Wallce C. H. Choy, for biography, see p. 92 of the January 1998 issue of this JOURNAL.

E. Herbert Li (S'87-M'88-SM'95), for photograph and biography, see p. 990 of the June 1998 issue of this JournaL. 